\title{
Poly(ADP-Ribose) Polymerase Inhibitor PJ34 Attenuated Hepatic Triglyceride Accumulation in Alcoholic Fatty Liver Disease in Mice ${ }^{\S}$
}

\author{
Shishun Huang, Bing Zhang, Yingli Chen, Huan Liu, Yang Liu, Xin Li, Zhiwei Bao, \\ Zhenyuan Song, and Zhigang Wang \\ College of Medical Laboratory Science and Technology, Harbin Medical University, Daqing, Heilongjiang, China (S.H., B.Z., Y.C., \\ Y.L., X.L., Z.B., Z.W.); Fuxin Center Hospital, Fuxin, Jilin, China (H.L.); and Department of Kinesiology and Nutrition, University of \\ Illinois, Chicago, Illinois (Z.S.)
}

Received May 30, 2017; accepted January 5, 2018

\section{ABSTRACT}

Poly(ADP-ribose) polymerase (PARP) is an NAD-consuming enzyme and its specific role in the pathogenesis of alcoholic fatty liver disease (AFLD) remains elusive. In this study, we applied PJ34 [N-(5,6-dihydro-6-oxo-2-phenanthridinyl)-2acetamide hydrochloride] to inhibit hepatic PARP activity to examine the corresponding pathologic alteration in AFLD in mice and the underlying molecular mechanism. We found that PJ34 decreased the intracellular triglyceride (TG) content in hepatocytes. Moreover, PJ34 suppressed the gene expression of diglyceride acyltransferases DGAT1 and DGAT2 and elevated intracellular $\mathrm{NAD}^{+}$levels in hepatocytes. These mechanistic observations were validated in alcohol-fed mice injected with PJ34 intraperitoneally. Our results indicate that the PJ34 injection attenuated hepatic TG accumulation in alcohol-fed mice. Furthermore, PJ34 injection lowered the gene expression of hepatic sterol regulatory element binding protein $1 \mathrm{c}$, DGAT1, and DGAT2, whereas PJ34 injection augmented hepatic $\mathrm{NAD}^{+}$levels in alcohol-fed mice. Finally, nicotinamide riboside supplementation alleviated hepatic TG accumulation in alcohol-fed mice. These data indicate that applying PARP-specific inhibitor PJ34 by intraperitoneal injection attenuated hepatic $\mathrm{NAD}^{+}$depletion and TG accumulation in alcohol-fed mice and may be a potential candidate for use in AFLD therapy.

\section{Introduction}

Alcoholic fatty liver disease (AFLD) is one of the most prevalent forms of chronic liver disease worldwide and has a widespread incidence. AFLD may progress to alcoholic liver disease, which is a major cause of illness and death in the United States (Purohit et al., 2004; Lívero and Acco, 2016). The pathogenesis of AFLD is not well established but is associated with excessive ethanol consumption, which causes hepatic lipid accumulation, enhances hepatic lipogenesis, and inhibits fatty acid oxidation (Rogers et al., 2008). AFLD can also progress to hepatitis, fibrosis, and cirrhosis, which may then lead to hepatocellular carcinoma and liver-related death.

This work was supported by the National Natural Science Foundation [Grant 81370523], a Postdoctoral Science Foundation Special Project [Grant 201104420], a China Postdoctoral Science Foundation General Project [Grant 20100471022], the Heilongjiang Young Key Academic Staff Support Program [Grant 1251G039], the Science and Technology Bureau of Daqing [Grant szd2015-02], and the Postgraduate Innovative Program [Grant YJSCX2015$59 \mathrm{HYD}]$.

https://doi.org/10.1124/jpet.117.243105.

S This article has supplemental material available at jpet.aspetjournals.org.
Nevertheless, the mechanisms of AFLD are complicated and are not completely understood.

Chronic alcohol consumption enhances lipogenesis and decreases fatty acid oxidation, which destroys the balance of intracellular fatty metabolism. Alcohol exposure induces the generation of reactive oxygen species, which causes oxidative stress and lipid accumulation in the liver (Roskams et al., 2003). Excessive alcohol consumption reduces the hepatic $\mathrm{NAD}^{+}$level, which disrupts the balance of the $\mathrm{NAD}^{+} / \mathrm{NADH}$ ratio and the elevation of the lactate/pyruvate ratio (Gao and Bataller, 2011). Ethanol metabolism mediates cellular redox status and disturbs several major hepatic lipid metabolism transcriptional regulators, such as sterol regulatory element binding protein 1c (SREBP-1c), lipin-1, AMP-activated kinase, peroxisome proliferator-activated receptor $\gamma$ coactivator 1- $\alpha$, and Forkhead box protein O1 (Harris and Finck, 2011; Everitt et al., 2013; You et al., 2015).

Poly(ADP-ribose) polymerase (PARP) is an abundant nuclear protein that plays key roles in a variety of cellular processes, including transcriptional regulation and DNA repair, and programmed cell death. PARP detects and initiates

ABBREVIATIONS: AF, alcohol-fed diet; AFLD, alcoholic fatty liver disease; ALT, alanine transaminase; AST, aspartate transaminase; DGAT, diglyceride acyltransferase; DMEM, Dulbecco's modified Eagle's medium; H\&E, hematoxylin and eosin; HFHS, high-fat high-sucrose; MK-4827, (S)-2-(4-(piperidin-3-yl)phenyl)-2H-indazole-7-carboxamide; NR, nicotinamide riboside; OA, oleic acid; pADPr, polymeric adenosine diphosphate ribose; PARP, poly(ADP-ribose) polymerase; PBS, phosphate-buffered saline; PCR, polymerase chain reaction; PF, pair-fed diet; PJ34, N-(5,6dihydro-6-oxo-2-phenanthridinyl)-2-acetamide hydrochloride; TC, total cholesterol; TG, triglyceride. 
an immediate cellular response to single-strand DNA breaks (by signaling DNA-repairing enzymes to repair the singlestrand DNA breaks). PARP is composed of four domains: a DNA-binding domain, a tryptophan-glycine-arginine-rich domain, an automodification domain, and a catalytic domain (Isabelle et al., 2010). PARP1 is the best-studied protein of the PARP family. Human PARP1 consists of six functional domains: three zinc finger DNA-binding domains (Zn1, Zn2, and $\mathrm{Zn} 3$ ), the automodification domain, the tryptophanglycine-arginine-rich domain, and the catalytic domain (Schreiber et al., 2006; Li et al., 2014). As one of the $\mathrm{NAD}^{+}$ consumers (Sirtuins, PARPs, and CD38), PARP catalyzes the synthesis of polymeric adenosine diphosphate ribose ( $\mathrm{pADPr}$ ) from $\mathrm{NAD}^{+}$to the target proteins, thereby regulating their activity (Kraus and Hottiger, 2013). Excessive hyperactivation of PARP causes the depletion of intercellular $\mathrm{NAD}^{+}$ and ATP levels, which leads to cellular metabolistic disorder and death. Inhibition of PARP activity promotes atherosclerotic plaque regression, attenuates high-fat diet-induced dysfunction of lipid metabolism, and alleviates diabetes (Hans et al., 2009; Zakaria et al., 2017). Chemical or genetic inhibition of PARP1 attenuates carbon tetrachloride-induced liver injury, inflammation, and fibrosis (Mukhopadhyay et al., 2014). Meanwhile, pharmacological inhibition of PARP1 reduces hepatocellular carcinoma growth (Quiles-Perez et al., 2010; Zhang et al., 2012). PARP1 knockout in mice protects against nonalcoholic steatohepatitis by restoring hepatic $\mathrm{NAD}^{+}$ content and sirtuin 1 activity (Mukhopadhyay et al., 2017; Ray, 2017), but the specific role of PARP in AFLD progression is still yet to be thoroughly investigated.

This study aimed to investigate the effect of the PARP inhibitor PJ34 [N-(5,6-dihydro-6-oxo-2-phenanthridinyl)-2acetamide hydrochloride; $\left.\mathrm{C}_{17} \mathrm{H}_{17} \mathrm{ClN}_{2} \mathrm{O}_{2}\right]$ on hepatic lipid accumulation induced by chronic alcohol feeding and its underlying mechanisms. We found that PJ34 attenuated hepatic $\mathrm{NAD}^{+}$depletion and triglyceride (TG) accumulation in both hepatocytes and alcohol-fed mice. Our results suggest that PARP inhibitor PJ34 could be applied as a potential therapeutic chemical treatment for AFLD by modulating intracellular $\mathrm{NAD}^{+}$levels.

\section{Materials and Methods}

Animal Care and Treatment. Male C57BL/6 mice (aged 8 weeks; mean \pm S.D. weight $20 \pm 0.5 \mathrm{~g}$ ) were obtained from the Harbin Medical University Experimental Animal Center (Daqing, Heilongjiang, China). All studies were approved by the Animal Ethical Committee of Harbin Medical University. The mice were housed in conventional conditions, were maintained on a 12-hour light/dark cycle (at $23 \pm 2{ }^{\circ} \mathrm{C}$ with $50 \% \pm 5 \%$ humidity), and were fed standard food and water ad libitum at the animal facility for 1 week before the experiment began. All mice were fed for 4 weeks with liquid diets. All diets were purchased from Research Diets Inc. (Trophic Animal Feed High-Tech, Nantong, China). The pair-fed diet (PF) (TP 4030A) contained 18\% protein, 35\% fat, and 47\% carbohydrate, whereas the alcohol-fed diet (AF) (TP 4030C) contained 18\% protein, $35 \%$ fat, $11 \%$ carbohydrate, and $36 \%$ alcohol as per LieberDeCarli (Fernando et al., 2013). Forty-five male C57BL/6 mice were then randomly divided into six groups and were fed respective diets for model development. The groups were as follows: 1) PF plus physiologic saline $(n=6), 2)$ PF plus PJ34 (Selleck Chemicals, Houston, TX), $(n=6)$, 3) PF plus nicotinamide riboside (NR) (Hangzhou LZ Chemical, Hangzhou, China) $(n=6)$, 4) AF plus physiologic saline $(n=9), 5) \mathrm{AF}$ plus PJ34 ( $n=9$ ), and 6) AF plus NR $(n=9)$. Treatment consisted of PJ34 as a PARP activity-specific inhibitor (10 mg/kg per day), which was injected intraperitoneally three times a week throughout the experiment. The same volume of physiologic saline $(0.1 \mathrm{ml} / 20 \mathrm{~g}$ per day i.p.) was injected intraperitoneally three times a week as a vehicle treatment. NR as a natural $\mathrm{NAD}^{+}$precursor $(10 \mathrm{mg} / \mathrm{kg}$ per day) was supplemented in the liquid diet throughout the experiment. Food consumption was recorded daily. Mice were weighed weekly on the same day. All animals were euthanized after an overnight fast. At the end of the experiment, plasma, liver samples, and epididymal fat pad samples were harvested.

Histologic Analysis. Liver samples were fixed in 4\% paraformaldehyde for 2 days, transferred to $70 \%$ ethanol, and dehydrated through a serial alcohol gradient. Tissues were embedded in a paraffin wax block and cut into $6-\mu \mathrm{m}$-thin sections. All sections were dewaxed in xylene, rehydrated through decreasing concentrations of ethanol, and washed in distilled water. Finally, tissue sections were stained with hematoxylin and eosin (H\&E). The fields were randomly selected and were photographed under $\times 400$ magnification. The quantitative statistics of lipid droplet fold change were analyzed with Image-Pro Plus software (Media Cybernetics, Rockville, MD).

Circulating Biochemical Measurement. Levels of circulating TG and total cholesterol (TC) were assessed with commercially available kits (BioSino, Beijing, China) as per the manufacturer's instructions. We took a certain amount of plasma into the working liquid in volume proportion (1:100). The mixture was incubated in a $37^{\circ} \mathrm{C}$ water bath for 10 minutes. Next, a SpectraMAX 190 instrument (Molecular Devices, Sunnyvale, CA) was used to detect the absorbance of TG and TC at a wavelength of $505 \mathrm{~nm}$. We used a commercially available enzyme-linked immunosorbent assay kit (Shanghai Jinma Laboratory Equipment, Shanghai, China) to measure alanine transaminase (ALT) and aspartate transaminase (AST) concentrations. The plasma was diluted five times and was incubated with a relative working liquid for 30 minutes in a $37^{\circ} \mathrm{C}$ incubator. Absorbance was detected by the SpectraMAX 190 instrument at a wavelength of $450 \mathrm{~nm}$. Concentrations of TG, TC, AST, and ALT were calculated with a related equation.

Hepatic TG and TC Measurement. Liver tissues were washed with phosphate-buffered saline (PBS) twice and $800 \mathrm{mg}$ liver tissue was homogenized in $1 \mathrm{ml} 50 \mathrm{mM} \mathrm{NaCl}$. Liver total lipids were extracted in a 7-ml heptane/isopropanol (3:2) mixture at room temperature in a shaker for 2 hours. The lipids were centrifuged at $3000 \mathrm{~g}$ for 5 minutes. The organic layer was collected in a new tube and dried in the hood. Liver TG and TC content was determined by the above-mentioned commercially available kit as described previously.

Cell Culture and Treatment. HepG2 cells, a human hepatoma cell line, were purchased from the Institute for Biologic Sciences (Shanghai, China). HepG2 cells were cultured in Dulbecco's modified Eagle's medium (DMEM) high glucose culture fluid (Gibco, Grand Island, NY) containing 10\% (v/v) fetal bovine serum supplemented with $2 \mathrm{mM}$ glutamine, $100 \mathrm{IU} / \mathrm{ml}$ penicillin, and $100 \mathrm{mg} / \mathrm{ml}$ streptomycin. The cells were incubated at $37^{\circ} \mathrm{C}$ in a humidified incubator with $5 \% \mathrm{CO}_{2}$ and $95 \%$ air. For pharmacological studies, cells were washed with PBS three times and replaced with a serum-free medium for serum starvation. After 12-hour culture, the cells were treated with PARP inhibitor PJ34 $(1 \mu \mathrm{M})$ in the absence or presence of $3.3 \mu \mathrm{M}$ oleic acid (OA) (Sigma, St. Louis, MO) for 24 hours. The same volume of serum-free medium acted as the control.

Intracellular TG Determination. After treatment with PJ34 in the presence OA for 24 hours, cells were harvested at the end of the experiment. For intracellular TG measurement, HepG2 cells were dissolved in $0.3 \mathrm{ml} \mathrm{NaOH}(50 \mathrm{mM})$ solution per well (24-well plate). Intracellular total lipids were extracted in $1 \mathrm{ml}$ heptane/isopropanol (3:2) mixture at room temperature in a shaker for 2 hours. The lipids were centrifuged at $3000 \mathrm{~g}$ for 5 minutes. The organic layer was placed in a new tube and dried in the hood. Intracellular TG content was determined by the above-mentioned commercially available kit as described previously. 
Hepatic and Intracellular $\mathrm{NAD}^{+}$Analysis. Liver tissue samples and intracellular $\mathrm{NAD}^{+}$levels were extracted with a commercially available kit (Suzhou Comin Biotechnology, Suzhou, China). Liver tissue samples and HepG2 cells were harvested after treatment. Five million cells were collected in the centrifugal tube and added to $1 \mathrm{ml}$ acid extract liquor. For the liver tissues, $0.1 \mathrm{~g}$ tissue was homogenized in $1 \mathrm{ml}$ acid extract liquor. The homogenized tissue was broken by ultrasonic for 1 minute in an ice bath, and then were bathed in $100^{\circ} \mathrm{C}$ for 5 minutes followed by cooling in ice bath and $10000 \mathrm{~g}$ centrifuge in $4^{\circ} \mathrm{C}$ for 10 minutes. The supernatant was placed in a new tube and the same volume of alkaline extract liquor was added before centrifugation at $10,000 \mathrm{~g}$ at $4^{\circ} \mathrm{C}$ for 10 minutes. The supernatant was used for testing as per the manufacturer's instructions. Finally, absorbance was detected with a SpectraMAX 190 instrument at a wavelength of $570 \mathrm{~nm}$. The $\mathrm{NAD}^{+}$concentration in each sample was calculated by a related equation.

Western Blot Analysis. Cells were washed three times with PBS and lysed in RIPA buffer (50 mM Tris, pH 7.4, $150 \mathrm{mM} \mathrm{NaCl}, 1 \%$ Triton X-100, 1\% sodium deoxycholate, 0.1\% SDS; Beyotime Biotechnology, Jiangsu, China) supplemented with phenylmethanesulfonyl fluoride (Beyotime Biotechnology) for 30 minutes. Liver tissues were ground with a glass homogenizer that was lysed in RIPA buffer. Whole cell extracts or tissue extracts were collected by centrifugation at $10,000 \mathrm{~g}$ at $4^{\circ} \mathrm{C}$ for 15 minutes. The protein concentration was determined using an Enhanced BCA Protein Assay Kit (Beyotime Biotechnology). Equal amounts of protein $(30 \mu \mathrm{g})$ were subjected to $10 \%$ or $8 \%$ SDS-PAGE depending on the molecular weight of the desired proteins and were transferred to a nitrocellulose transfer membrane (Pall Corporation, New Port Richey, FL). The membranes were blocked with $5 \%$ powdered milk diluted in Tris-buffered saline with $0.05 \%$ Tween 20 for 1 hour at room temperature and were incubated with primary antibodies at $4^{\circ} \mathrm{C}$ overnight. The primary antibodies used were mouse monoclonal IgG3 anti-pADPr $(10 \mathrm{H}$; Santa Cruz Biotechnology, Dallas, TX), rabbit monoclonal antiPARP (46D11; Cell Signaling Technology, Danvers, MA), rabbit polyclonal anti-SREBP-1c (Bioss, Beijing, China), mouse monoclonal anti-SREBP-1 (Santa Cruz Biotechnology), rabbit polyclonal anti-diglyceride acyltransferase DGAT1 (Bioss), rabbit polyclonal anti-DGAT2 antibody (Bioss), and mouse monoclonal anti- $\beta$-actin (ZSGB-BIO, Beijing, China). The antigen-primary antibody complexes were washed and then incubated with horseradish peroxidase-conjugated secondary antibodies at room temperature for 1 hour. Goat anti-mouse and anti-rabbit horseradish peroxidase-conjugated secondary antibodies were used (ZSGB-BIO). Peroxidase was detected using enhanced chemiluminescence Western blotting detection reagents (HaiGene, Harbin, China). Densitometric analysis was performed using Quantity One software (Bio-Rad, Hercules, CA).

RNA Extraction and Real-Time Polymerase Chain Reaction Assays. Total RNAs were extracted from liver tissues or HepG2 cells. Total RNAs were isolated using TRIzol reagent (HaiGene) as per the manufacturer's manual. For each sample, $1 \mu \mathrm{g}$ total RNA was reverse transcribed using a Golden First cDNA Synthesis Kit (HaiGene) as per the manufacturer's instructions $\left(30^{\circ} \mathrm{C}\right.$ for $15 \mathrm{~min}$ utes, $55^{\circ} \mathrm{C}$ for 50 minutes, and $95^{\circ} \mathrm{C}$ for 10 minutes). Real-time polymerase chain reaction (PCR) of SREBP-1c, DGAT1, DGAT2, and 18s rRNA mRNA was performed in an Applied Biosystems 7300 real-time PCR system (Applied Biosystems, Foster City, CA) by using SYBR Green qPCR Mix (Roche, Basel, Switzerland) under the following conditions: $95^{\circ} \mathrm{C}$ for 10 minutes, 45 cycles of $95^{\circ} \mathrm{C}$ for 10 seconds, $58^{\circ} \mathrm{C}$ for 20 seconds, and $72^{\circ} \mathrm{C}$ for 30 seconds. To amplify the target genes, all primers were purchased from GenScript Biotechnology (Nanjing, China). Quantitative normalization of the cDNA in each sample was performed using the 18s rRNA gene as an internal control. Real-time PCR assays were performed in duplicate for each sample, and the mean value was used for the calculation of mRNA expression levels.
Statistical Analysis. Statistical analysis was performed using one-way analysis of variance and the Newman-Keuls test with OriginPro7.5 (OriginLab, Austin, TX) and Graph Pad Prism (version 5; Graph Pad Software Inc., La Jolla, CA) software. Data are presented as means \pm S.E. $P$ values $<0.05$ were considered to indicate a statistically significant difference.

\section{Results}

Chronic Alcohol Consumption Induced Hepatic Fat Accumulation and Liver Injury in Mice. C57BL/6 mice were fed a Lieber-DeCarli alcohol-containing diet for 4 weeks. Liver samples were harvested for histologic examination and the hepatic TG content was detected. As shown in our results, chronic alcohol feeding significantly decreased body weight and the epididymal fat pad weight/body weight rate (Fig. 1, A and C) compared with the $\mathrm{PF}$ group, in which liver weight/body weight rate significantly increased (Fig. 1B). Chronic alcohol consumption increased hepatic TG content and circulating TG levels (Fig. 1, F and G). Chronic alcohol exposure increased circulating ALT, AST, and TC levels (Fig. 1, D, E, and I) and hepatic TC content (Fig. 1H). The severity of AFLD was analyzed with $\mathrm{H} \& \mathrm{E}$ staining (Fig. $1, \mathrm{~J}$ and $\mathrm{K}, \times 400$ ). In the $\mathrm{AF}$ group, the lobular structures were destroyed and widely distributed, with more lipid droplets compared with the PF group. The data showed that chronic alcohol consumption induced hepatic lipid accumulation and liver injury.

Chronic Alcohol Feeding Enhanced PARP Expression, pADPr Synthesis, and NAD ${ }^{+}$Depletion in Mice. Mice were fed an ethanol-containing liquid diet for 4 weeks; liver samples were then harvested for further experiments. Total proteins were extracted from the liver samples. Western blot analysis was used to detect PARP expression and activation in alcohol-fed mice. Our results showed that chronic alcohol feeding elevated PARP protein expression (Fig. 2, A and B) and activity, which was shown by higher pADPr conjugated with proteins (Fig. 2, A and C), compared with the $\mathrm{PF}$ group. Furthermore, chronic alcohol consumption decreased hepatic $\mathrm{NAD}^{+}$levels (Fig. 2D) and increased gene expression and protein abundance in TG anabolism, such as SREBP-1c, DGAT1, and DGAT2 (Fig. 2, E-J), compared with the $\mathrm{PF}$ group. The SREBP-1 precursor $(125 \mathrm{kDa})$ and mature SREBP-1 (68 kDa) were significantly increased in alcohol-fed mice and PJ34 injection reduced SREBP-1 expression and activation compared with the AF group (Supplemental Fig. 7). Thus, our results indicated that chronic alcohol consumption-induced hepatic TG accumulation was associated with hepatic PARP overexpression and activation and subsequent hepatic $\mathrm{NAD}^{+}$ depletion.

Inhibition of PARP Activity Increased $\mathrm{NAD}^{+}$Levels and Lowered Intracellular TG Content in Hepatocytes. HepG2 cells were used to investigate whether PARP inhibition modulated hepatic lipid accumulation and the underlying mechanisms. Our results showed that 24-hour PJ34 exposure $(1 \mu \mathrm{M})$ decreased pADPr synthesis (Fig. 3C) but had no effect on the protein abundance of PARP (Fig. 3, A and B) in hepatocytes. Furthermore, we found that PJ34 elevated intracellular $\mathrm{NAD}^{+}$ content (Fig. 3D), whereas PJ34 treatment lowered intracellular TG content (Fig. 3E). These results demonstrated that PARP inhibition augmented intracellular $\mathrm{NAD}^{+}$and decreased intracellular TG accumulation in HepG2 cells. 
A

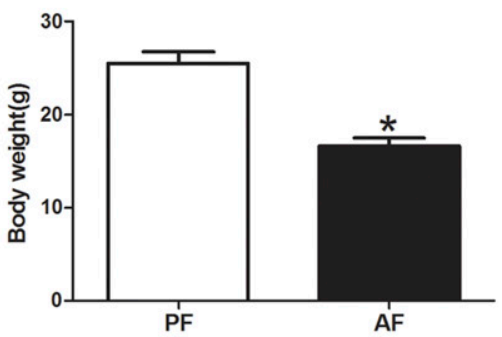

D

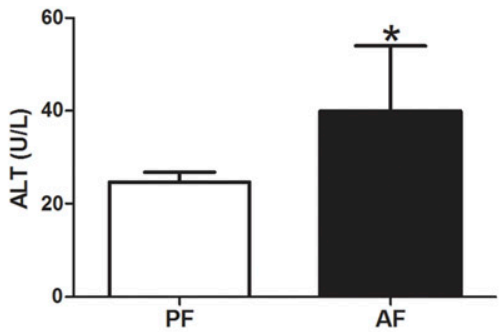

G

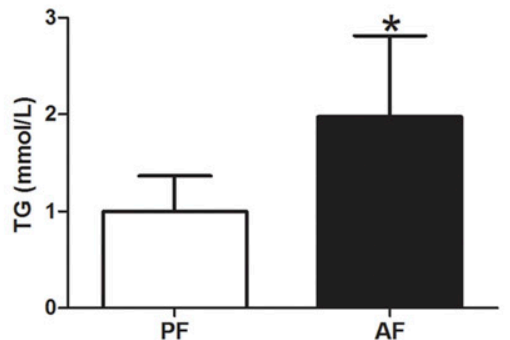

$\mathbf{J}$

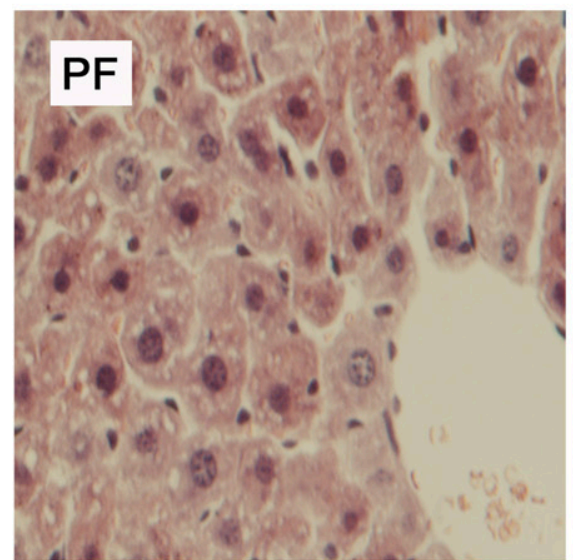

B

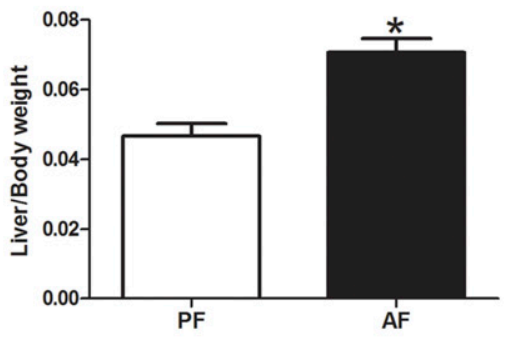

$\mathbf{E}$

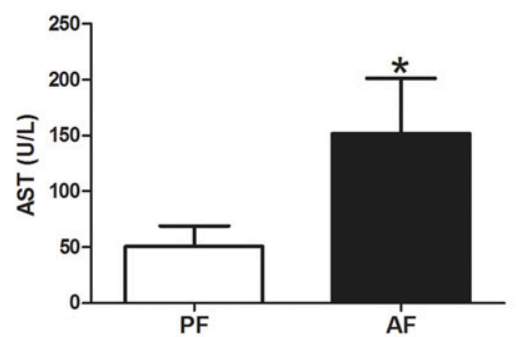

H
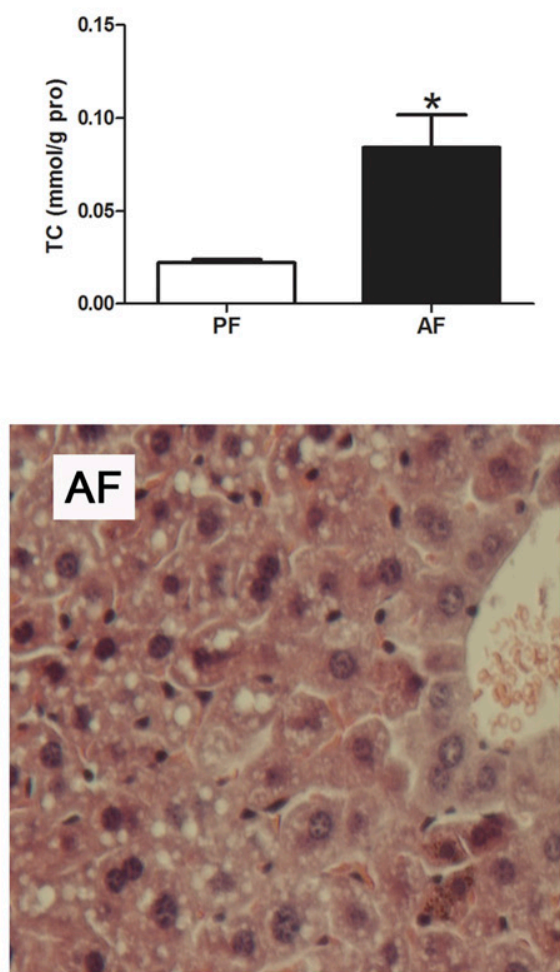

C

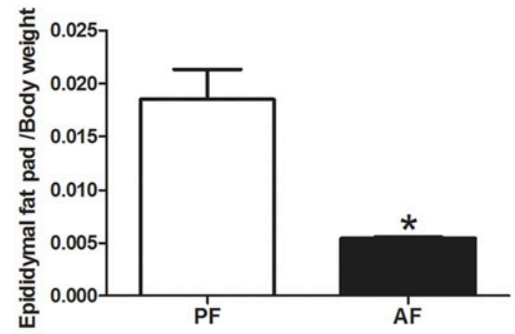

$\mathbf{F}$

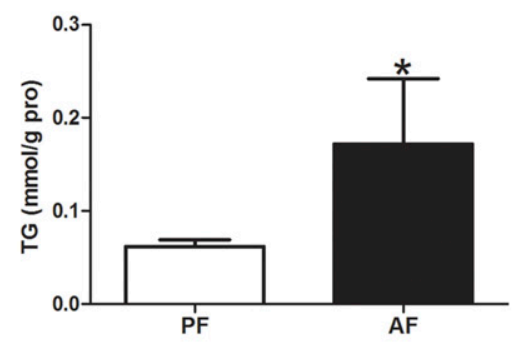

I

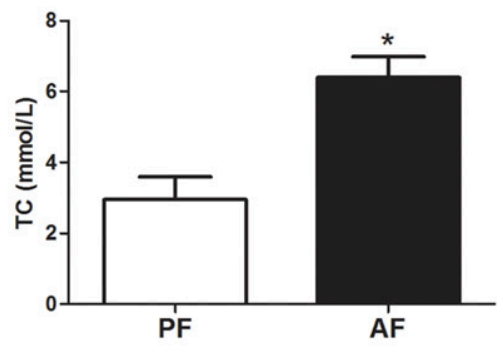

$\mathbf{K}$

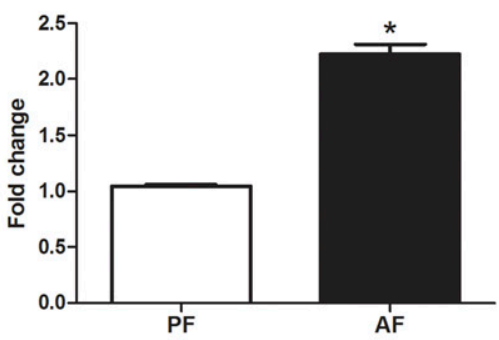

Fig. 1. Chronic alcohol consumption induced hepatic fat accumulation and liver injury in C57BL/6 mice. Mice were fed a Lieber-DeCarli alcoholcontaining diet for 4 weeks. (A and B) Chronic alcohol feeding lowered body weight (A) and increased liver/body weight rate (B) compared with the PF group. (C) The epididymal fat pad/body weight rate also decreased through chronic alcohol feeding. (D-I) Chronic alcohol consumption significantly increased levels of circulating ALT (D), AST (E), hepatic TG (F), circulating TG (G), hepatic TC (H), and TC (I). (J) Chronic alcohol feeding enhanced hepatic fat accumulation, as shown by H\&E staining of liver sections. (K) Quantitative statistics of lipid droplet fold change are shown. Values are means \pm S.D. ( $n=6$ per group). $* P<0.05$ (significant differences compared with the PF group). Original magnification, $\times 400$ in $(J)$.

Inhibition of PARP Activity Suppressed the Expression of Genes in TG Anabolism. To gain insight into the mechanism underlying PJ34-decreased intracellular TG accumulation, HepG2 cells were exposed to PJ34 $(1 \mu \mathrm{M})$ in complete DMEM with OA addition for 24 hours. The critical genes of TG anabolism were detected by real-time PCR. We found that PJ34 treatment significantly lowered the gene expression of DGAT1 and DGAT2 (Fig. 4, B and $\mathrm{C}$ ), but the gene expression of SREBP-1c changed little (Fig. 4A) in HepG2 cells. Consistent with the 
A

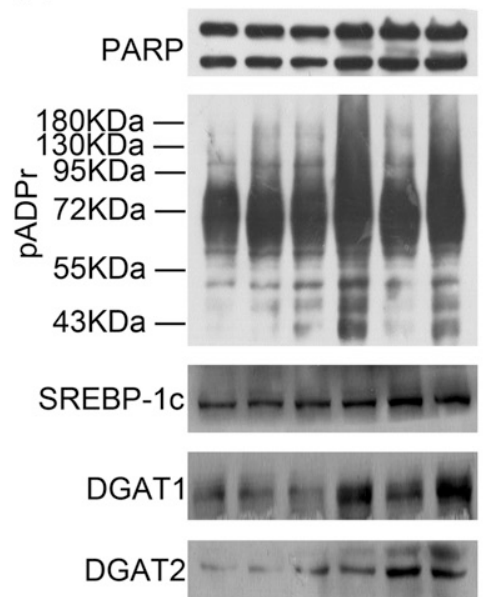

$\beta$-actin

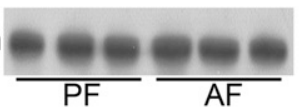

B

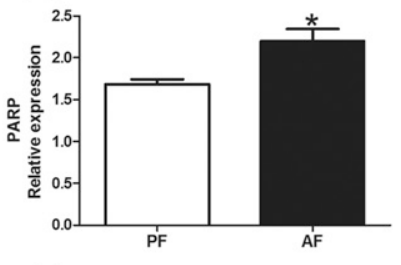

E

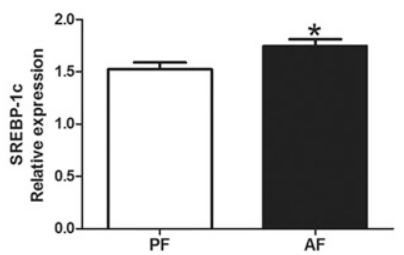

H

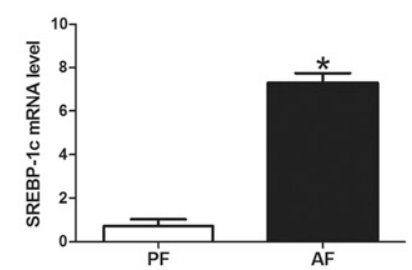

C

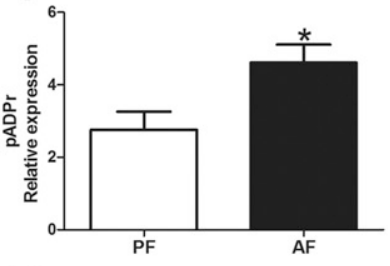

F

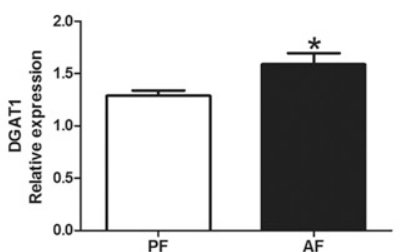

I

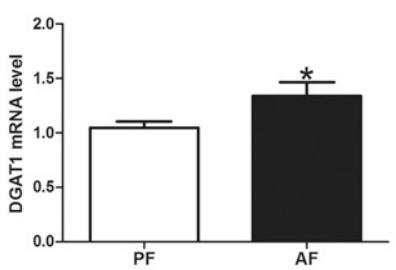

D

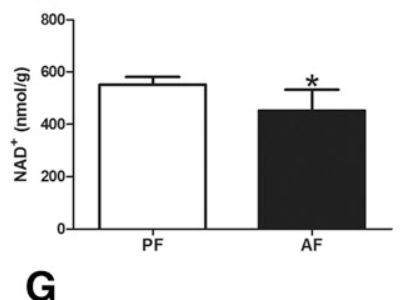

G

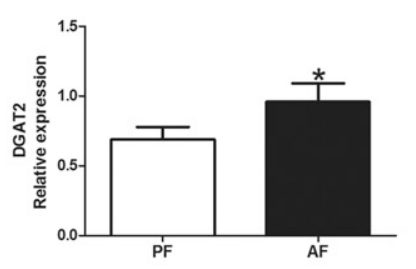

$\mathbf{J}$

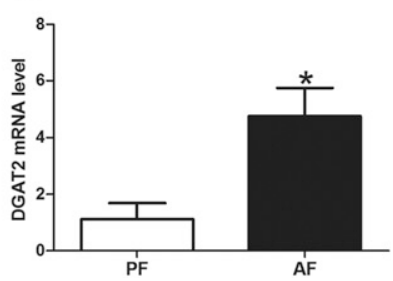

Fig. 2. Chronic alcohol feeding enhanced PARP expression, pADPr synthesis, and $\mathrm{NAD}^{+}$depletion in alcohol-fed mice. (A) Total proteins were extracted from liver tissues and subjected to Western blot analysis for expression of PARP, pADPr, SREBP-1c, DGAT1, and DGAT2 with specific antibodies. (B-D) Chronic alcohol exposure increased the expression of hepatic PARP protein (B), which catalyzed pADPr formation $(\mathrm{C})$ and lowered hepatic NAD ${ }^{+}$content compared with the PF group (D). (E-G) Densitometric analysis showed that chronic alcohol feeding increased the expression of SREBP-1c (E), DGAT1 (F), and DGAT2 $(\mathrm{G})$ proteins. (H-J) Relative gene expression of SREBP-1c (H), DGAT1 (I), and DGAT2 (J) was significantly higher in mice with chronic alcohol consumption compared with the PF group. Values are means \pm S.D. $(n=6$ per group). $* P<0.05$ (significant differences compared with the PF group).

PJ34-triggered elevation of intracellular TG content (Fig. $3 \mathrm{E})$, our results demonstrated that applying PJ34 to inhibit PARP activity decreased TG accumulation, and the underlying mechanism was involved in PJ34- inhibited gene expression of DGAT1 and DGAT2 in TG anabolism in HepG2 cells.

PJ34 Injection Attenuated Hepatic TG Accumulation in AF Mice. To detect the specific role of PARP inhibition in

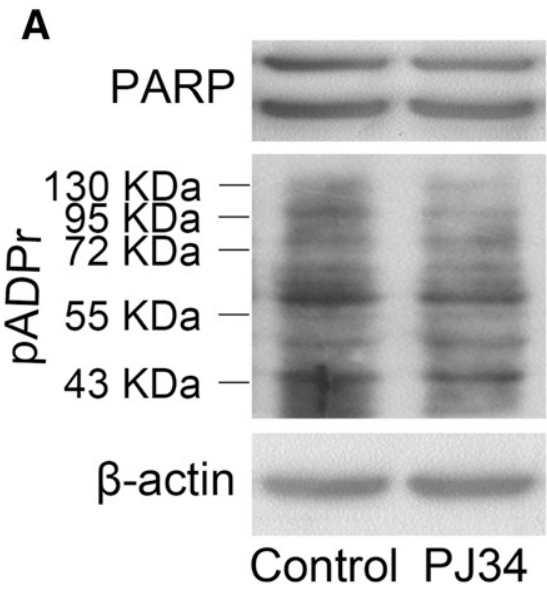

B

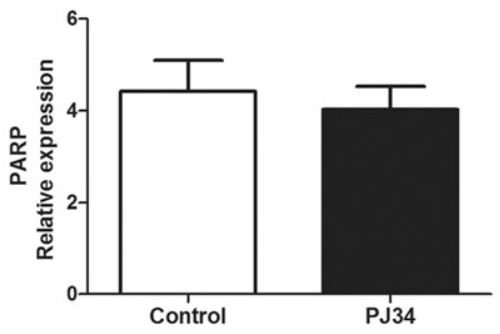

D

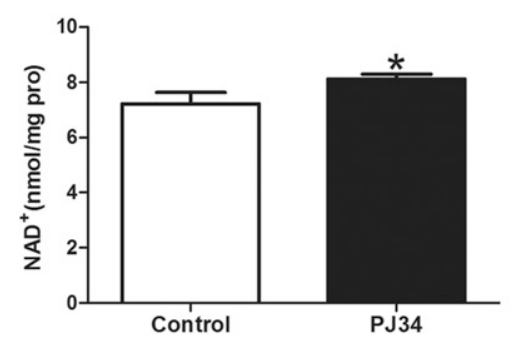

C

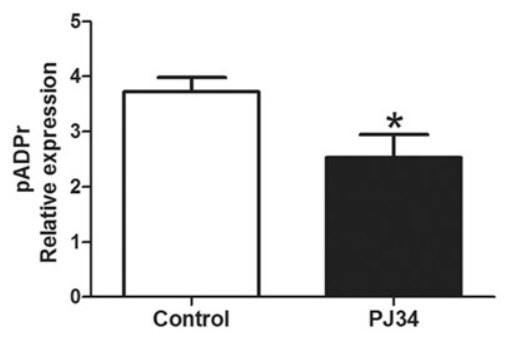

E

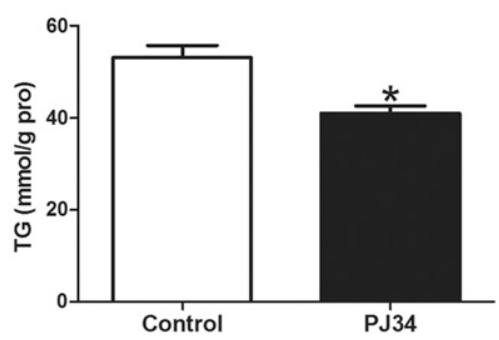

Fig. 3. Inhibition of PARP activity augmented $\mathrm{NAD}^{+}$levels and decreased intracellular TG content in hepatocytes. HepG2 cells were exposed to complete DMEM containing PJ34 $(1 \mu \mathrm{M})$ for 24 hours. (A) Expression of intracellular PARP and pADPr was detected by Western blot analysis. (B and C) PJ34 treatment did not significantly change the expression of PARP (B) and decreased the synthesis of pADPr (C). (D) PJ34 treatment elevated intracellular $\mathrm{NAD}^{+}$content. (E) Moreover, PJ34 treatment also decreased intracellular TG content. Values are means \pm S.D. from three or more independent batches of cells. $* P<0.05$ (compared with the control group). 
A

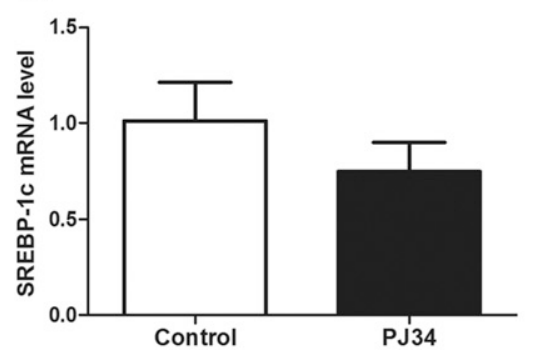

B

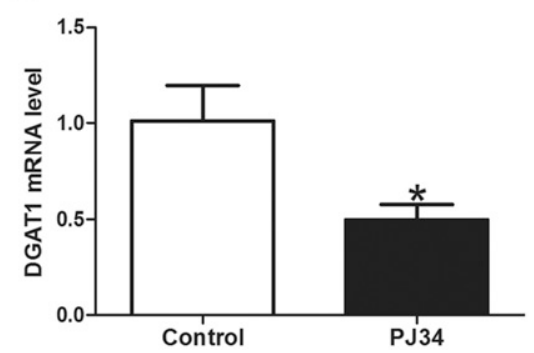

C

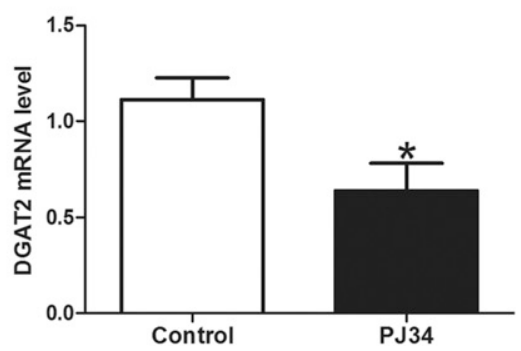

Fig. 4. Inhibition of PARP activity suppressed the expression of critical genes in TG anabolism. HepG2 cells were treated with PJ34 (1 $\mu \mathrm{M})$ in the presence of OA for 24 hours. Total RNAs were extracted from cells. The mRNA of critical genes was detected by real-time PCR. (A-C) PJ34 treatment changed little in intracellular SREBP-1c (A) and lowered intracellular gene expression of DGAT1 (B) and DGAT2 (C). Values are means \pm S.D. from three or more independent batches of cells. ${ }^{*} P<0.05$ (compared with control group).

the pathogenesis of AFLD, C57BL/6 mice were exposed to an alcohol-containing liquid diet with or without PJ34 intraperitoneal injection for 4 weeks. As shown in our results, PJ34 injection increased body weight (Fig. 5A) and decreased liver weight/body weight rate (Fig. 5B) in comparison to the $\mathrm{AF}$ group, but there were no significant changes in epididymal fat pad weight/body weight rate (Fig. 5C) compared with the AF group. Moreover, PJ34 injection decreased hepatic TG content and circulating TG levels compared with the AF group (Fig. 5, F and G). In our results, PJ34 injection had no role in hepatic TC content and circulating TC and ALT levels compared with the AF group (Fig. 5, D, H, and I). However, PJ34 injection significantly decreased circulating AST levels compared with the AF group (Fig. 5E). In addition, H\&E staining showed that PJ34 injection alleviated hepatic lipid accumulation and the lobular structures were more intact in the AF plus PJ34 group compared with the AF group (Fig. 5, J and K).

PJ34 Injection Elevated Hepatic $\mathrm{NAD}^{+}$Levels and Lowered Gene Expression in TG Anabolism in AlcoholFed Mice. To detect the underlying mechanism for PJ34alleviated TG accumulation in the pathogenesis of AFLD, C57BL/6 mice were fed liquid diets containing alcohol with PJ34 injection for 4 weeks. We found that PJ34 injection suppressed hepatic PARP activation by decreasing the level of pADPr compared with the AF group (Fig. 6, A-C). PJ34 injection alleviated hepatic alcohol-induced $\mathrm{NAD}^{+}$depletion compared with the AF group (Fig. 6D). Furthermore, the expression of critical genes, including SREBP-1c, DGAT1, and DGAT2, was significantly lowered by PJ34 injection compared with the AF group (Fig. 6, E-G). Our data demonstrated that PJ34 injection alleviated hepatic TG accumulation via increasing $\mathrm{NAD}^{+}$content and inhibiting TG anabolism in the liver in alcohol-fed mice.

NR Supplementation Attenuated Hepatic TG Accumulation in Alcohol-Fed Mice. To further demonstrate the specific role of $\mathrm{NAD}^{+}$in TG anabolism, C57BL/6 mice were fed an alcohol-containing liquid diet supplemented with NR for 4 weeks. We found that NR supplementation increased body weight (Fig. 7A) and decreased liver weight/body weight rate (Fig. 7B) compared with the AF group. No significant change was observed in epididymal fat pad weight/body weight rate (Fig. 7C) and hepatic TC content (Fig. 7E) in alcohol-fed mice after NR supplementation. Importantly, NR supplementation elevated hepatic $\mathrm{NAD}^{+}$content $(\mathrm{Fig} .7 \mathrm{~F})$ and alleviated hepatic TG accumulation (Fig. 7D) in alcohol-fed mice. Our data suggested that elevating hepatic $\mathrm{NAD}^{+}$ content by NR supplementation attenuated chronic alcohol consumption-induced hepatic TG accumulation.

\section{Discussion}

In this study, we demonstrate that PARP inhibitor PJ34 alleviated TG accumulation in both hepatocytes and the liver of alcohol-fed mice; the underlying mechanism was involved in PJ34-elevated $\mathrm{NAD}^{+}$levels and PJ34-inhibited gene expression of SREBP-1c, DGAT1, and DGAT2 in the liver of alcohol-fed mice. Our data suggest that PJ34 had therapeuticc potential in AFLD by increasing hepatic $\mathrm{NAD}^{+}$ content and decreasing hypertriglyceridemia and hepatic TG accumulation.

Various publications show that PARP has been gaining recognition as a central regular signaling molecule in numerous diseases, including cancer, energetic metabolism, inflammation, shock, cardiovascular diseases, diabetes, and fatty liver disease (Sistigu et al., 2015; Huang et al., 2017; Islam et al., 2017; Vida et al., 2017). High-fat high-sucrose (HFHS) diets lead to insulin resistance, oxidative stress, steatosis, and inflammation and lower hepatic $\mathrm{NAD}^{+}$levels, driving reductions in hepatic mitochondrial dysfunction and increases in hepatic lipid content in mice (Verbeek et al., 2015; Gariani et al., 2016). HFHS diets also increase PARP activity in mice, as shown by the ADP-ribose polymerization of proteins catalyzed by PARPs. $\mathrm{NAD}^{+}$was used to produce ADP-ribose for PARP-catalyzed ADP-ribose polymerization, followed by reduced intracellular $\mathrm{NAD}^{+}$levels, which disrupted mitochondrial function and the resulting hepatic lipid accumulation (Gariani et al., 2017). Consistent with these investigations, we found that chronic alcohol feeding caused PARP overactivation and higher levels of ADP-ribose polymers of proteins in mice compared with the PF group. This suggested that PARP activation was involved in the pathogenesis of AFLD. As a $\mathrm{NAD}^{+}$consumer, PARP activation promoted the accumulation of ADP-ribose polymers and $\mathrm{NAD}^{+}$depletion in many diseases (Banasik et al., 2012; Shetty et al., 2014; Martire et al., 2015; Rappou et al., 2016). $\mathrm{NAD}^{+}$depletion impaired cellular redox capacity and metabolic transformation (Mouchiroud et al., 2013; Yu et al., 2016). In our investigation, chronic alcohol feeding for 4 weeks caused the decline of hepatic $\mathrm{NAD}^{+}$levels resulting from hepatic PARP activation. Prior studies reported that $\mathrm{NAD}^{+}$ overconsumption leads to the metabolic disorder of fatty acids and subsequent hepatic lipid accumulation (Rappou et al., 2016; Mukhopadhyay et al., 2017). Increasing evidence showed that the alteration of PARP expression and activity was associated with lipid metabolism (Bai and Csóka, 2015; 
A

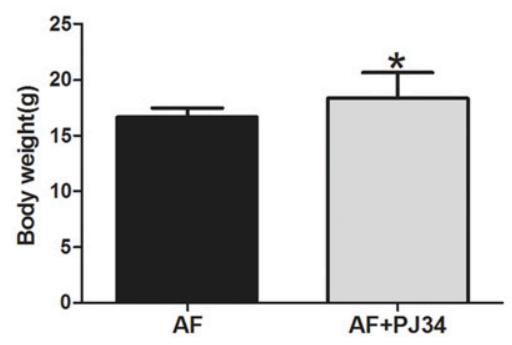

D

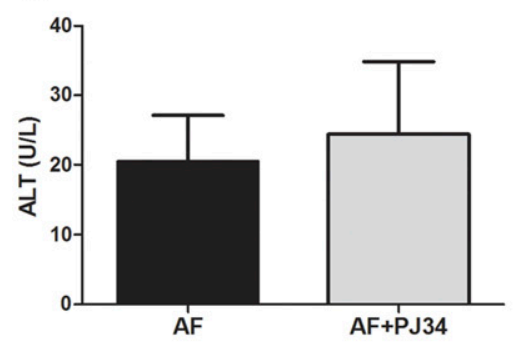

G

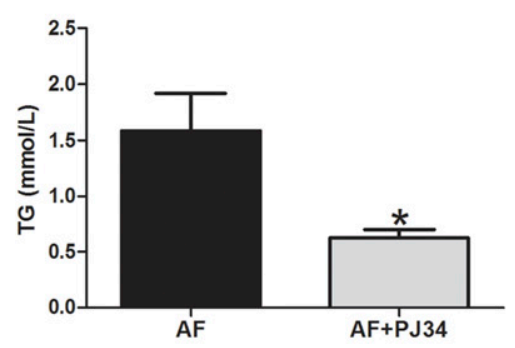

J

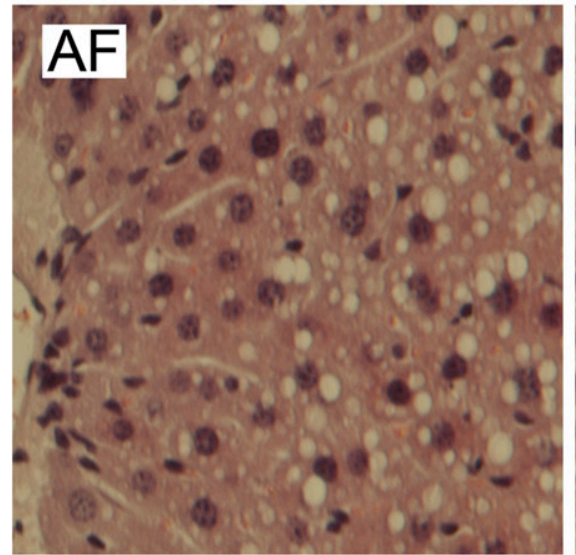

B

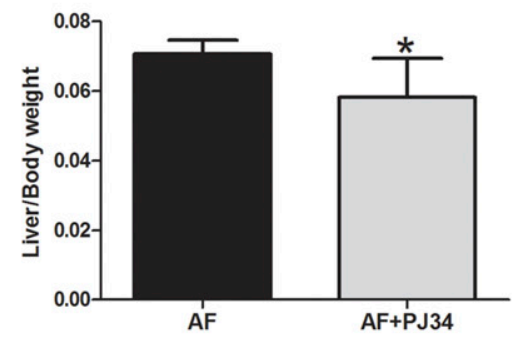

E

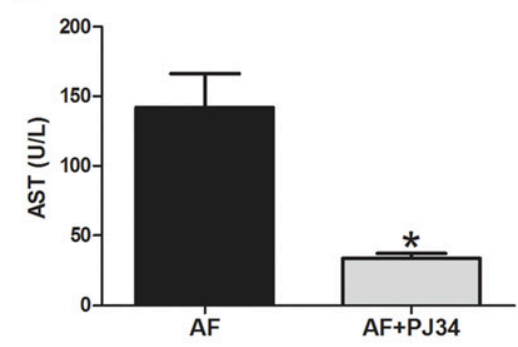

H
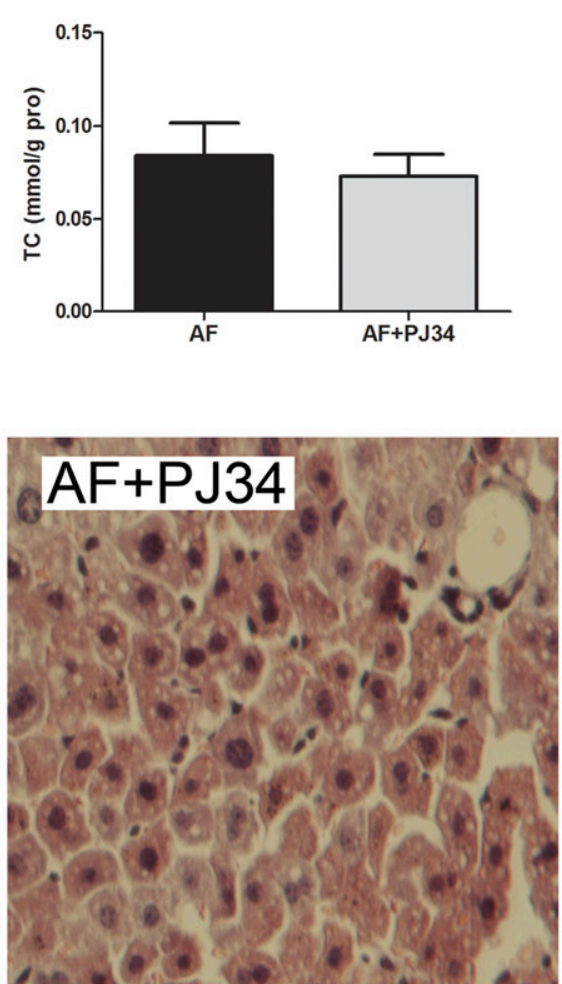

C

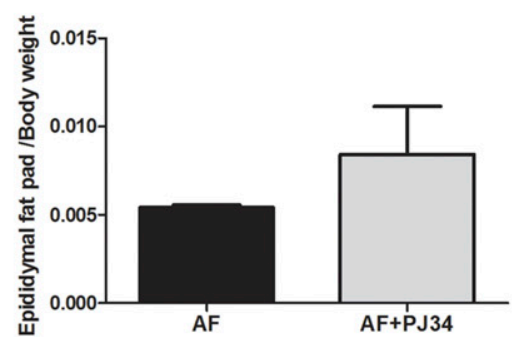

$\mathbf{F}$

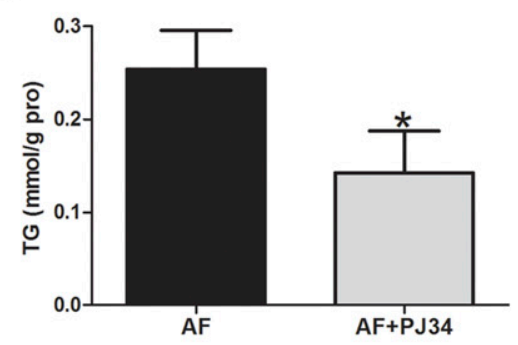

I

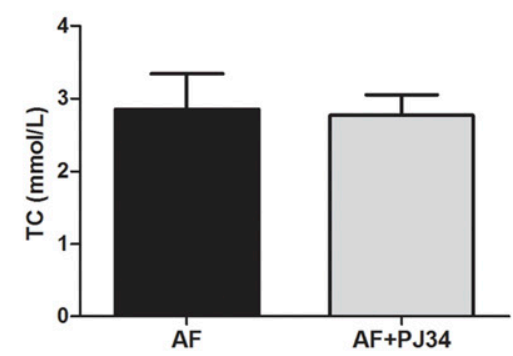

K

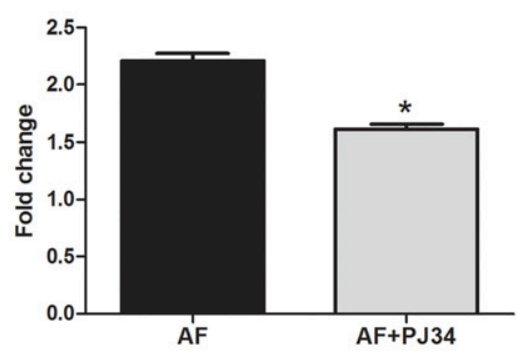

Fig. 5. PJ34 injection attenuated hepatic TG accumulation in alcohol-fed mice. C57BL/6 mice were fed an alcohol-containing liquid diet with or without PJ34 intraperitoneal injection for 4 weeks. (A and B) Compared with the AF group, PJ34 injection augmented body weight (A) and lowered the liver/body weight rate (B). (C-E) However, PJ34 injection did not significantly change the epididymis fat/body weight rate (C) and circulating ALT and AST levels (D and E). (F-I) PJ34 intraperitoneal injection decreased hepatic TG content (F) and circulating TG levels (G) but had no difference on hepatic TC content $(\mathrm{H})$ and the level of circulating TC (I) compared with the AF group. (J) Liver sections were stained with H\&E. (K) The quantitative statistics of lipid droplet fold change are shown. Values are means \pm S.D. ( $n=6$ per group). $* P<0.05$ (compared with the AF group). Original magnification, $\times 400$ in $(\mathrm{J})$.

Kiss et al., 2015; Pang et al., 2015). Therefore, both the data and literature indicate that chronic alcohol consumption-induced activation of hepatic PARP and the decline of hepatic $\mathrm{NAD}^{+}$content have critical roles in hepatic TG accumulation. Thus, the pharmacological modulation of hepatic PARP activity might be a potential candidate for use in AFLD therapy.

Similar considerations have been seen in several recent reports. PARP inhibitor olaparib (4-[[3-[[4-(cyclopropylcarbonyl)-1piperazinyl]carbonyl]-4-fluorophenyl]methyl]-1(2H)-phthalazinone; 


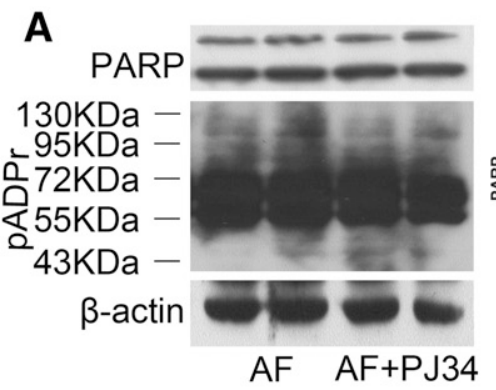

B C

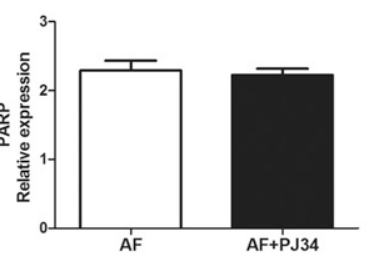

E

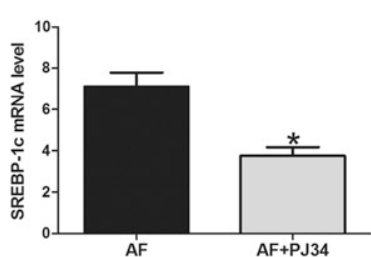

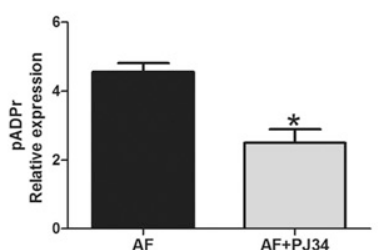

D

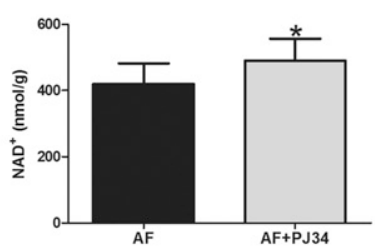

G

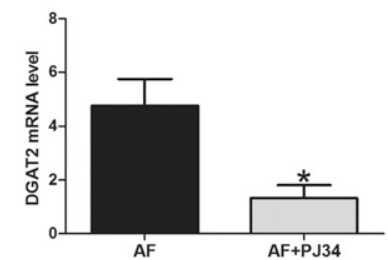

Fig. 6. PJ34 injection elevated hepatic NAD ${ }^{+}$levels and lowered gene expression in TG anabolism in alcohol-fed mice. Male C57BL/6 mice were fed alcohol-containing liquid diets and injected intraperitoneally with or without PJ34 for 4 weeks. (A) Total proteins were extracted from liver tissues and were used to detect PARP expression and pADPr synthesis with specific antibodies. (B and C) PJ34 injection had no effect on PARP expression (B) and decreased pADPr synthesis (C). (D) PJ34 injection reduced hepatic $\mathrm{NAD}^{+}$depletion compared with the AF group. (E-G) PJ34 injection significantly alleviated hepatic critical genes expression of SREBP-1 (E), DGAT1 (F), and DGAT2 (G) compared with the AF group. Values are means \pm S.D. $(n=6$ per group). $* P<0.05$ (compared with the $\mathrm{AF}$ group).

$\mathrm{C}_{24} \mathrm{H}_{23} \mathrm{FN}_{4} \mathrm{O}_{3}$ ) improved hepatic fatty acid oxidation and NAFLD induced by a HFHS diet (Gariani et al., 2017). Pharmacological inhibition of PARP or genetic deletion of PARP1 can restore hepatic $\mathrm{NAD}^{+}$content and increase SIRT1 activation, which decreases hepatic TG accumulation in both alcoholic and nonalcoholic steatohepatitis (Mukhopadhyay et al., 2017). In our study, we found that PJ34 injection for 4 weeks lowered both circulating TG and hepatic
TG levels in alcohol-fed mice, but no significant changes were observed for circulating and hepatic TC levels in the same conditions. Mechanistic investigations in HepG2 cells also showed that PJ34 significantly decreased intracellular TG levels; however, there was little change in PARP expression levels in PJ34-treated cells. Furthermore, PJ34 reduced the gene expression of DGAT1 and DGAT2, both of which are key enzymes in TG anabolism. Nevertheless, there was no
A

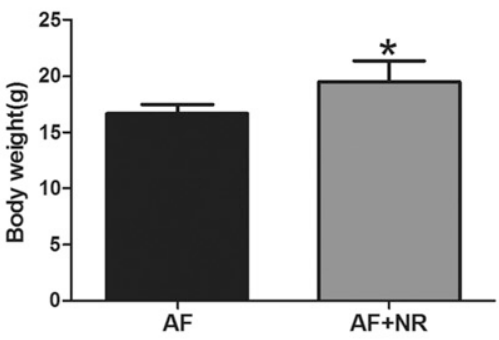

D

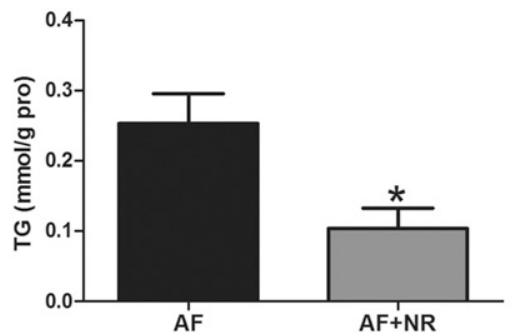

B

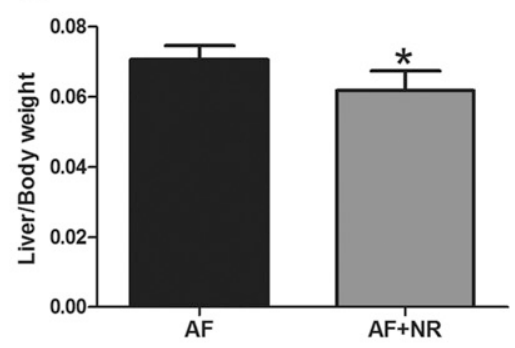

E

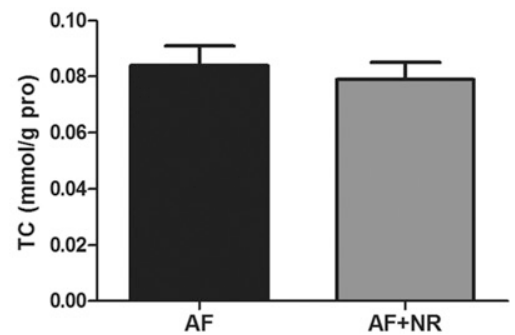

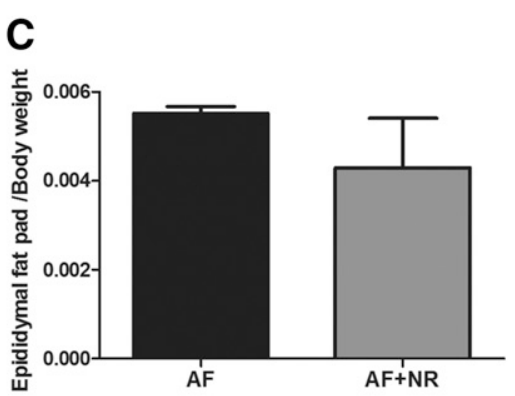

F

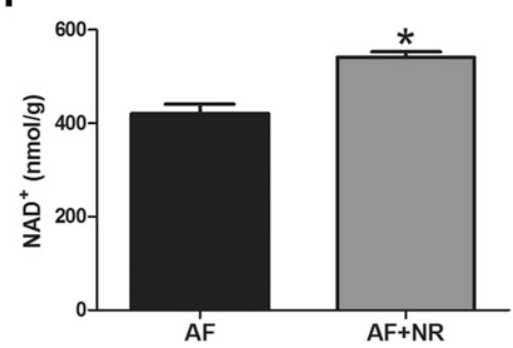

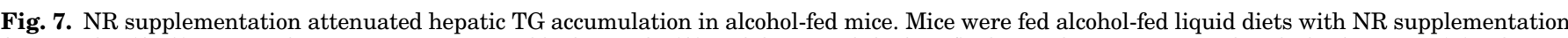

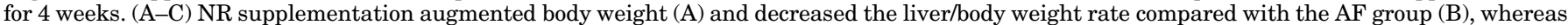

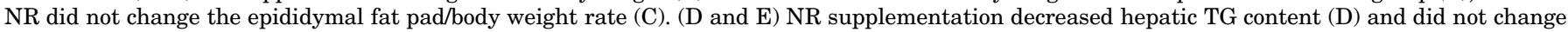

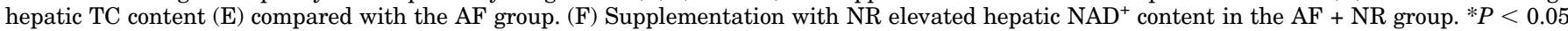
(significant differences compared with the AF group). 
significant change in the gene expression of SREBP-1c, the lipogenic transcriptional factor in PJ34-treated hepatocytes, compared with the control group. Whether the transcriptional activity of SREBP-1c was lowered by PJ34 exposure needs to be confirmed in further studies. All of the mechanistic observations in hepatocytes were validated in alcohol-fed mice. PJ34 injection for 4 weeks decreased hepatic gene expression of SREBP-1c, DGAT1, and DGAT2 in alcohol-fed mice. Overall, our results suggested that PJ34 attenuated hepatic TG accumulation in AFLD progression and the underlying mechanism was involved in the PJ34-suppressed gene expression of DGAT1 and DGAT2 in TG anabolism.

PARP2 is another isoform of PARP. PARP1 and PARP2 act as a metabolic regulator by interacting with many transcription factors regulating lipid metabolism and oxidation (Bai and Cantó, 2012). In the liver, PARP2 deletion or inhibition enhanced SIRT1 expression and promoted mitochondrial biogenesis in mice (Bai et al., 2011). Hepatic cholesterol levels were higher in $\mathrm{PARP}^{-/-}$mice. PARP2 deletion or inhibition increased hepatic cholesterol homeostasis and lowered highdensity lipoprotein levels in plasma via enhanced SREBP-1 expression (Szántó et al., 2014). In our experiments, PJ34 injection could not change hepatic and plasma TC levels in alcohol-fed mice (Fig. 5, H and I). Our data indicate that PJ34, as a chemical inhibitor of activity of both PARP1 and PARP2, might exert differentiated roles between PARP1 and PARP2 in hepatic lipid metabolism, which must be confirmed in further investigation.

Since hepatic PARP-related $\mathrm{NAD}^{+}$alteration was associated with the dysfunction of TG accumulation in the progression of nonalcoholic steatohepatitis (Gariani et al., 2017; Mukhopadhyay et al., 2017), we aimed to demonstrat whether it accounted for progression of alcoholic steatohepatitis. In our study, chronic alcohol feeding significantly decreased hepatic $\mathrm{NAD}^{+}$levels compared with the PF group. In addition, PARP inhibitor PJ34 injections elevated hepatic $\mathrm{NAD}^{+}$levels in alcohol-fed mice. Data from the HepG2 cell lines also showed that PJ34 elevated intracellular $\mathrm{NAD}^{+}$content. All changes in hepatic $\mathrm{NAD}^{+}$by PARP inhibition were associated with hepatic TG alteration. To further demonstrate PARPregulated $\mathrm{NAD}^{+}$in alcoholic hepatic TG metabolism, we supplemented NR in the alcohol diet for 4 weeks simultaneously. Recent studies have shown that $\mathrm{NR}$ as a $\mathrm{NAD}^{+}$ precursor increases $\mathrm{NAD}^{+}$in both skeletal muscle and the liver, which enhances oxidation metabolism and protects against high-fat diet-induced metabolic dysfunction via activated sirtuin 1 and sirtuin 3 (Cantó et al., 2012). In our study, we found that NR supplementation increased body weight and lowered liver weight in alcohol-fed mice. NR supplementation elevated hepatic $\mathrm{NAD}^{+}$content and attenuated hepatic TG accumulation in alcohol-fed mice. Our data suggested that a high level of $\mathrm{NAD}^{+}$supplementation alleviated alcoholic hepatic $\mathrm{NAD}^{+}$depletion and TG accumulation in the progression of AFLD. A low level of PARP activation-induced hepatic $\mathrm{NAD}^{+}$might be one reason for the pathogenesis of AFLD.

PARP inhibitors approved by the U.S. Food and Drug Administration and European Medicines Agency are currently being tested in clinical trials (Parkes and Kennedy, 2016). A phase III trial with the PARP1 and PARP2 inhibitor niraparib [MK-4827; (S)-2-(4-(piperidin-3-yl)phenyl)-2Hindazole-7-carboxamide] for ovarian cancer showed that niraparib inhibited PARP-triggered DNA damage and cell death and ultimately oncogenic progression (Kanjanapan et al., 2017; Lin and Kraus, 2017). Another oral PARP inhibitor, olaparib, is undergoing phase III trials for patients with ovarian cancer (Ledermann, 2016b) and results demonstrate that olaparib prolonged survival in some patients with advanced ovarian cancer (Ledermann et al., 2016a). This inhibitor was also used for the treatment of breast and colorectal cancer and showed promising results (Robert et al., 2017). In vivo, C57BL/6 mice were fed alcoholcontaining liquid diets and were injected intraperitoneally with or without olaparib or rucaparib [8-fluoro-5-(4((methylamino)methyl)phenyl)-3,4-dihydro-2 $H$-azepino[5,4,3$c d$ indol-1 $(6 H)$-one phosphoric acid, $\left.\mathrm{C}_{19} \mathrm{H}_{18} \mathrm{FN}_{3} \mathrm{O}_{5} \mathrm{P}\right]$ for 4 weeks. Olaparib and rucaparib injection attenuated hepatic fat droplet accumulation in alcohol-fed mice (Supplemental Fig. 1). Both drugs attenuated hepatic TG accumulation, decreased circulating TG and TC levels, and reduced liver injury as a result of chronic alcoholic consumption in alcoholfed mice (Supplemental Fig. 2). In addition, we found that injection with olaparib or rucaparib elevated hepatic $\mathrm{NAD}^{+}$ levels in alcohol-fed mice and attenuated hepatic $\mathrm{NAD}^{+}$ depletion by chronic alcoholic exposure (Supplemental Fig. $3)$. The same results were found in alcohol-fed mice that received treatment with PJ34. In vitro, HepG2 cells were exposed to complete DMEM medium with or without $\mathrm{H}_{2} \mathrm{O}_{2}$ (an activator of $\mathrm{PARP}) . \mathrm{H}_{2} \mathrm{O}_{2}$ reduced intracellular $\mathrm{NAD}^{+}$levels and enhanced intracellular TG content in hepatocytes. PARP activity by $\mathrm{H}_{2} \mathrm{O}_{2}$ enhanced the expression of critical genes in TG anabolism in HepG2 cells (Supplemental Fig. 4). Moreover, PARP inhibition by PJ34 attenuated hepatic TG accumulation and inflammation (Supplemental Fig. 5) in mice who received chronic alcohol feeding. Similarly, chronic alcohol feeding increased the expression of proinflammatory cytokines interleukin-6, monocyte chemoattractant protein-1, and tumor necrosis factor- $\alpha$. Olaparib, rucaparib, or PJ34 injection markedly attenuated liver inflammation in liver tissues assessed by immunohistochemical methods (Supplemental Fig. 6). These results suggest that PARP inhibition might be a promising therapeutic strategy for alcoholic steatohepatitis. Our data indicated that PARP inhibitor (olaparib, rucaparib or PJ34) injection significantly elevated hepatic $\mathrm{NAD}^{+}$levels and attenuated hepatic TG accumulation in alcohol-fed mice.

In general, our investigation provides primary evidence that the exogenous PARP pharmacological inhibitor PJ34 is effective against alcohol-induced hepatic TG accumulation by enhancing the hepatic $\mathrm{NAD}^{+}$content and suppressing the gene expression of DGAT1 and DGAT2 in the liver in alcoholfed mice. Thus, the chemical inhibitor PJ34 has therapeutic potential in AFLD progression by restoring hepatic $\mathrm{NAD}^{+}$ depletion and lowering hepatic TG anabolism.

\section{Authorship Contributions}

Participated in research design: Huang, Song, Wang.

Conducted experiments: Huang, Zhang, Chen, Wang.

Contributed new reagents or analytic tools: Zhang, H. Liu, Y. Liu, Li, Bao.

Performed data analysis: Chen, H. Liu, Y. Liu, Li, Bao.

Wrote or contributed to the writing of the manuscript: Huang, Song, Wang.

\section{References}

Bai P and Cantó C (2012) The role of PARP-1 and PARP-2 enzymes in metabolic regulation and disease. Cell Metab 16:290-295. 
Bai P, Canto C, Brunyánszki A, Huber A, Szántó M, Cen Y, Yamamoto H, Houten SM, Kiss B, Oudart H, et al. (2011) PARP-2 regulates SIRT1 expression and wholebody energy expenditure. Cell Metab 13:450-460.

Bai P and Csóka B (2015) New route for the activation of poly(ADP-ribose) polymerase-1: a passage that links poly(ADP-ribose) polymerase-1 to lipotoxicity? Biochem J 469:e9-e11.

Banasik M, Stedeford T, and Strosznajder RP (2012) Natural inhibitors of poly(ADPribose) polymerase-1. Mol Neurobiol 46:55-63.

Cantó C, Houtkooper RH, Pirinen E, Youn DY, Oosterveer MH, Cen Y, FernandezMarcos PJ, Yamamoto H, Andreux PA, Cettour-Rose P, et al. (2012) The NAD $(+)$ precursor nicotinamide riboside enhances oxidative metabolism and protects against high-fat diet-induced obesity. Cell Metab 15:838-847.

Everitt H, Hu M, Ajmo JM, Rogers CQ, Liang X, Zhang R, Yin H, Choi A, Bennett ES, and You M (2013) Ethanol administration exacerbates the abnormalities in hepatic lipid oxidation in genetically obese mice. Am J Physiol Gastrointest Liver Physiol 304:G38-G47.

Fernando H, Wiktorowicz JE, Soman KV, Kaphalia BS, Khan MF, and Shakeel Ansari GA (2013) Liver proteomics in progressive alcoholic steatosis. Toxicol Appl Pharmacol 266:470-480.

Gao B and Bataller R (2011) Alcoholic liver disease: pathogenesis and new therapeutic targets. Gastroenterology 141:1572-1585.

Gariani K, Menzies KJ, Ryu D, Wegner CJ, Wang X, Ropelle ER, Moullan N, Zhang H, Perino A, Lemos V, et al. (2016) Eliciting the mitochondrial unfolded protein response by nicotinamide adenine dinucleotide repletion reverses fatty liver disease in mice. Hepatology 63:1190-1204.

Gariani K, Ryu D, Menzies KJ, Yi HS, Stein S, Zhang H, Perino A, Lemos V, Katsyuba E, Jha P, et al. (2017) Inhibiting poly ADP-ribosylation increases fatty acid oxidation and protects against fatty liver disease. J Hepatol 66:132-141.

Hans CP, Zerfaoui M, Naura AS, Troxclair D, Strong JP, Matrougui K, and Boulares AH (2009) Thieno[2,3-c]isoquinolin-5-one, a potent poly(ADP-ribose) polymerase inhibitor, promotes atherosclerotic plaque regression in high-fat diet-fed apolipoprotein E-deficient mice: effects on inflammatory markers and lipid content. $J$ Pharmacol Exp Ther 329:150-158.

Harris TE and Finck BN (2011) Dual function lipin proteins and glycerolipid metabolism. Trends Endocrinol Metab 22:226-233.

Huang K, Du M, Tan X, Yang L, Li X, Jiang Y, Wang C, Zhang F, Zhu F, Cheng M, et al. (2017) PARP1-mediated PPAR $\alpha$ poly(ADP-ribosyl)ation suppresses fatty acid oxidation in non-alcoholic fatty liver disease. J Hepatol 66:962-977.

Isabelle M, Moreel X, Gagné JP, Rouleau M, Ethier C, Gagné P, Hendzel MJ, and Poirier GG (2010) Investigation of PARP-1, PARP-2, and PARG interactomes by affinity-purification mass spectrometry. Proteome Sci 8:22.

Islam BU, Habib S, Ali SA, Moinuddin, and Ali A (2017) Role of peroxynitrite-induced activation of poly(ADP-ribose) polymerase (PARP) in circulatory shock and related pathological conditions. Cardiovasc Toxicol 17:373-383.

Kanjanapan Y, Lheureux S, and Oza AM (2017) Niraparib for the treatment of ovarian cancer. Expert Opin Pharmacother 18:631-640.

Kiss B, Szántó M, Szklenár M, Brunyánszki A, Marosvölgyi T, Sárosi E, Remenyik É, Gergely P, Virág L, Decsi T, et al. (2015) Poly(ADP) ribose polymerase-1 ablation alters eicosanoid and docosanoid signaling and metabolism in a murine model of contact hypersensitivity. Mol Med Rep 11:2861-2867.

Kraus WL and Hottiger MO (2013) PARP-1 and gene regulation: progress and puzzles. Mol Aspects Med 34:1109-1123.

Ledermann JA, Harter P, Gourley C, Friedlander M, Vergote I, Rustin G, Scott C, Meier W, Shapira-Frommer R, Safra T, et al. (2016a) Overall survival in patients with platinum-sensitive recurrent serous ovarian cancer receiving olaparib maintenance monotherapy: an updated analysis from a randomised, placebo-controlled, doubleblind, phase 2 trial. Lancet Oncol 17:1579-1589.

Ledermann JA (2016b) PARP inhibitors in ovarian cancer. Ann Oncol 27 (Suppl 1):i40-i44.

Li X, Erden O, Li L, Ye Q, Wilson A, and Du W (2014) Binding to WGR domain by salidroside activates PARP1 and protects hematopoietic stem cells from oxidative stress. Antioxid Redox Signal 20:1853-1865.

Lin KY and Kraus WL (2017) PARP inhibitors for cancer therapy. Cell 169:183.

Lívero FA and Acco A (2016) Molecular basis of alcoholic fatty liver disease: from incidence to treatment. Hepatol Res 46:111-123.

Martire S, Mosca L, and d'Erme M (2015) PARP-1 involvement in neurodegeneration: a focus on Alzheimer's and Parkinson's diseases. Mech Ageing Dev 146-148:53-64.

Mouchiroud L, Houtkooper RH, and Auwerx J (2013) NAD ${ }^{+}$metabolism: a therapeutic target for age-related metabolic disease. Crit Rev Biochem Mol Biol 48 $397-408$
Mukhopadhyay P, Horváth B, Rajesh M, Varga ZV, Gariani K, Ryu D, Cao Z, Holovac E, Park O, Zhou Z, et al. (2017) PARP inhibition protects against alcoholic and nonalcoholic steatohepatitis. J Hepatol 66:589-600.

Mukhopadhyay P, Rajesh M, Cao Z, Horváth B, Park O, Wang H, Erdelyi K, Holovac E, Wang Y, Liaudet L, et al. (2014) Poly (ADP-ribose) polymerase-1 is a key mediator of liver inflammation and fibrosis. Hepatology 59:1998-2009.

Pang J, Cui J, Gong H, Xi C, and Zhang TM (2015) Effect of NAD on PARP-mediated insulin sensitivity in oleic acid treated hepatocytes. J Cell Physiol 230:1607-1613. Parkes EE and Kennedy RD (2016) Clinical application of poly(ADP-ribose) polymerase inhibitors in high-grade serous ovarian cancer. Oncologist 21:586-593.

Purohit V, Russo D, and Coates PM (2004) Role of fatty liver, dietary fatty acid supplements, and obesity in the progression of alcoholic liver disease: introduction and summary of the symposium. Alcohol 34:3-8.

Quiles-Perez R, Muñoz-Gámez JA, Ruiz-Extremera A, O’Valle F, Sanjuán-Nuñez L, Martín-Alvarez AB, Martín-Oliva D, Caballero T, Muñoz de Rueda P, León J, et al. (2010) Inhibition of poly adenosine diphosphate-ribose polymerase decreases hepatocellular carcinoma growth by modulation of tumor-related gene expression. Hepatology 51:255-266.

Rappou E, Jukarainen S, Rinnankoski-Tuikka R, Kaye S, Heinonen S, Hakkarainen A, Lundbom J, Lundbom N, Saunavaara V, Rissanen A, et al. (2016) Weight loss is associated with increased NAD(+)/SIRT1 expression but reduced PARP activity in white adipose tissue. J Clin Endocrinol Metab 101:1263-1273.

Ray K (2017) Steatohepatitis: PARP inhibition protective against alcoholic steatohepatitis and NASH. Nat Rev Gastroenterol Hepatol 14:3.

Robert M, Frenel JS, Gourmelon C, Patsouris A, Augereau P, and Campone M (2017) Olaparib for the treatment of breast cancer. Expert Opin Investig Drugs 26: 751-759.

Rogers CQ, Ajmo JM, and You M (2008) Adiponectin and alcoholic fatty liver disease. IUBMB Life 60:790-797.

Roskams T, Yang SQ, Koteish A, Durnez A, DeVos R, Huang X, Achten R, Verslype C, and Diehl AM (2003) Oxidative stress and oval cell accumulation in mice and humans with alcoholic and nonalcoholic fatty liver disease. Am J Pathol 163: 1301-1311.

Schreiber V, Dantzer F, Ame JC, and de Murcia G (2006) Poly(ADP-ribose): novel functions for an old molecule. Nat Rev Mol Cell Biol 7:517-528.

Shetty PK, Galeffi F, and Turner DA (2014) Nicotinamide pre-treatment ameliorates $\mathrm{NAD}(\mathrm{H})$ hyperoxidation and improves neuronal function after severe hypoxia Neurobiol Dis 62:469-478.

Sistigu A, Manic G, Obrist F, and Vitale I (2015) Trial watch - inhibiting PARP enzymes for anticancer therapy. Mol Cell Oncol 3:e1053594.

Szántó M, Brunyánszki A, Márton J, Vámosi G, Nagy L, Fodor T, Kiss B, Virág L, Gergely P, and Bai P (2014) Deletion of PARP-2 induces hepatic cholesterol accumulation and decrease in HDL levels. Biochim Biophys Acta 1842:594-602.

Verbeek J, Lannoo M, Pirinen E, Ryu D, Spincemaille P, Vander Elst I, Windmolders P, Thevissen K, Cammue BP, van Pelt J, et al. (2015) Roux-en-y gastric bypass attenuates hepatic mitochondrial dysfunction in mice with non-alcoholic steatohepatitis. Gut 64:673-683.

Vida A, Márton J, Mikó E, and Bai P (2017) Metabolic roles of poly(ADP-ribose) polymerases. Semin Cell Dev Biol 63:135-143.

You M, Jogasuria A, Taylor C, and Wu J (2015) Sirtuin 1 signaling and alcoholic fatty liver disease. Hepatobiliary Surg Nutr 4:88-100.

Yu JH, Song SJ, Kim A, Choi Y, Seok JW, Kim HJ, Lee YJ, Lee KS, and Kim JW (2016) Suppression of PPAR $\gamma$-mediated monoacylglycerol O-acyltransferase 1 expression ameliorates alcoholic hepatic steatosis. Sci Rep 6:29352.

Zakaria EM, El-Maraghy NN, Ahmed AF, Ali AA, and El-Bassossy HM (2017) PARP inhibition ameliorates nephropathy in an animal model of type 2 diabetes: focus on oxidative stress, inflammation, and fibrosis. Naunyn Schmiedebergs Arch Pharmacol 390:621-631.

Zhang JX, Li DQ, He AR, Motwani M, Vasiliou V, Eswaran J, Mishra L, and Kumar R (2012) Synergistic inhibition of hepatocellular carcinoma growth by cotargeting chromatin modifying enzymes and poly (ADP-ribose) polymerases. Hepatology 55: $1840-1851$.

Address correspondence to: Dr. Zhigang Wang, College of Medical Laboratory Science and Technology, Harbin Medical University, No. 39, Xinyang Road, Gaoxin District, Daqing, Heilongiiang 163319, China. E-mail: wangzhigang@hmudq.edu.cn 